\title{
EXIGENCIAS DE MACRONUTRIENTES DE DOIS CULTIVARES DE MANDIOCA (Manihot esculenta CRANTZ) *
}

\author{
J.O. LORENZI $* *$ \\ J.R. GALLO $* *$ \\ E. MALAVOLTA $* * *$
}

\section{RESUMO}

As exigências de $\mathrm{N}, \mathrm{P}, \mathrm{K}, \mathrm{Ca}, \mathrm{Mg}$ e $\mathrm{S}$ dos cultivares de mandioca (Branca de Santa Catarina e IAC Mantiqueira) foram avalia das através da análise de material colh $\bar{i}$ do em ensaio de campo. Verificou-se difé rença apenas quanto à quantidade de $\mathrm{P}$ ex traîdo e $\mathrm{K}$ e $\mathrm{S}$ exportado (raízes).

\section{INTRODUÇÃO}

Com cerca de trinta milhões de toneladas anuais, o Brasil ocupa o primeiro lugar em produção de mandioca representando aproximadamente $30 \%$ da produção mundial (NESTEL, 1973). Ela é produzida em todos os estados da federação e em volume

* Parte da dissertação de Mestrado do primeiro autor, com auxílio do CNPq. Entregue para publicação em $24 / 10 /$ 1980 .

** Instituto Agronômico, Campinas, S. Paulo.

*** Departamento de Química, E.S.A "Luiz de Queiroz", USP. 
de produção, depois da cana-de-açúcar, é a mais importante do Paîs (IBGE, 1975). Embora o rendimento nacional de 12,6 t/ha seja maior que a média anual (9,1 t/ha, FAO, 1975), é forçoso reconhecer que a mandioca é uma das culturas de mais baixa ậlicação tecnológicas.

A Iiteratura mostra que a mandioca retira do solo quan tidades relativamente grandes de minerais, principalmente de potássio; no entanto, no Brasil, é prática corrente cultivála nos solos mais pobres, mesmo sem adubação, onde ela pode competir favoravelmente com outras culturas.

Na Tabela 1 são apresentadas as amplitudes de variação da extração de nutrientes pela planta inteira correspondentes a produção de 1 (uma) tonelada de raízes e da extração de nutrientes somente pelas raizes, ou seja, as quantidades exportadas. Estes valores são oriundos dos dados obtidos da 1 i teratura (Tabelas 2 e 3) que, para facilidade de comparação, foram transformados para quilos por tonelada de raizes.

Tabela 1 - Extração e exportação de nutrientes por uma tonelada de raízes de mandioca, em kg

\begin{tabular}{lrccc}
\hline Elementos & Extração & Média & Exportação & Média \\
\hline Nitrogênio & $19,93-20,10$ & 6,78 & $0,70-6,85$ & 2,22 \\
Fósforo & $6,67-2,40$ & 1,33 & $0,29-0,77$ & 0,48 \\
Potássio & $4,69-14,96$ & 7,28 & $1,77-7,14$ & 3,51 \\
Cálcio & $1,00-9,90$ & 3,51 & $0,28-1,34$ & 0,66 \\
Magnésio & $0,46-2,20$ & 1,19 & $0,05-1,08$ & 0,39 \\
\hline
\end{tabular}

Verificaram-se diferenças acentuadas nos dados obtidos pelos diversos autores, as quais são devidas, principalmente a terem trabalhado com cultivares diferentes e condições va riadas de clima, solo e tratos culturais.

O presente trabalho teve por objetivo verificar as necessidades nutricionais e suas possiveis diferenças entre o 


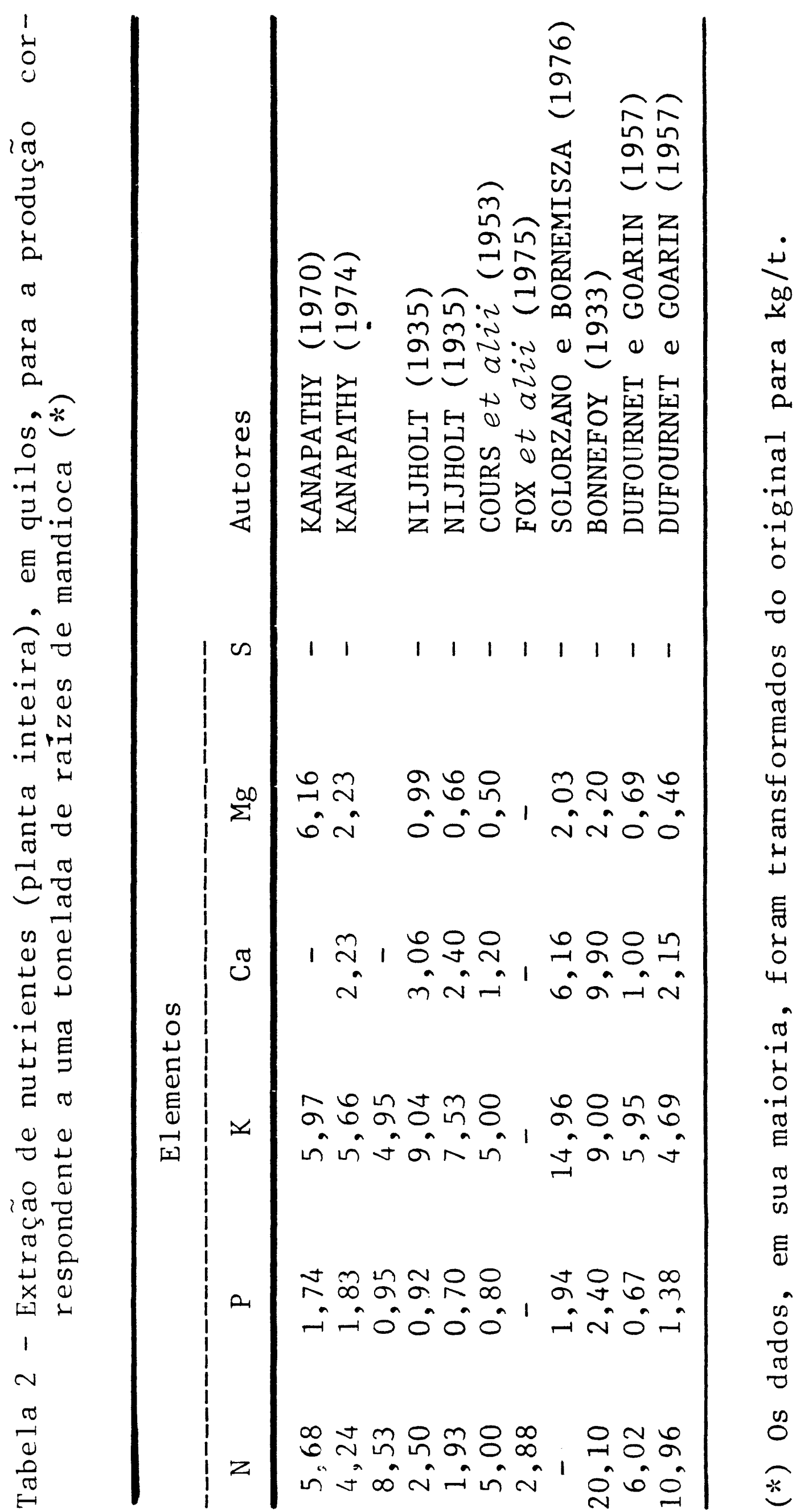




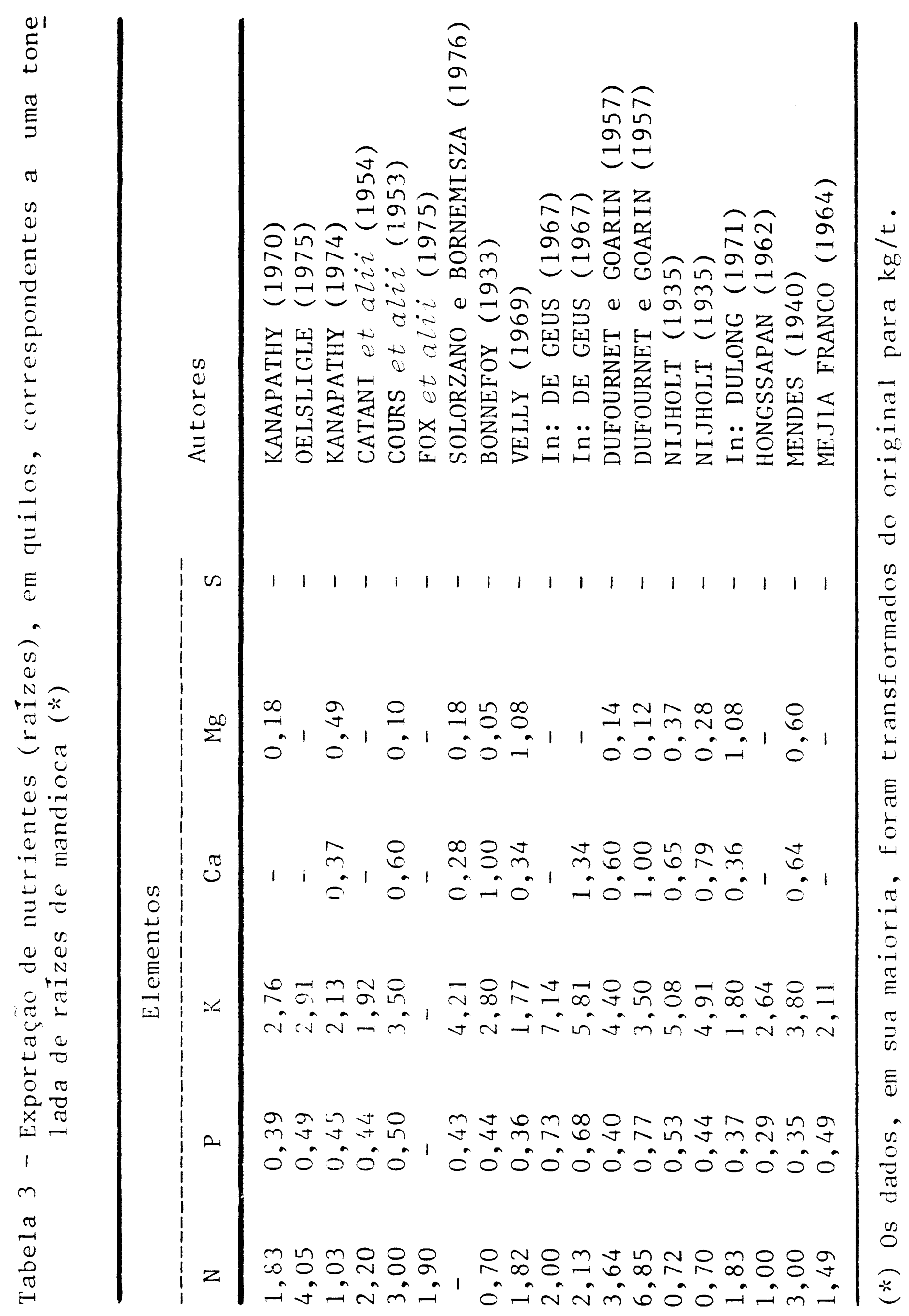


cultivar IAC Mantiqueira (mandioca mansa) e o cultivar Branca de Santa Catarina (mandioca brava ou tóxica), nos seguintes aspectos:

a) extração e exportação dos macronutrientes:

b) produção de raỉzes.

\section{MATERIAL E METTODOS}

Foram utilizados os cultivares de mandioca (Manihotesculenta Crantz) Branca de Santa Catarina e IAC Mantiqueira; o primeiro foi introduzido e, o segundo, criado e selecionado pela Seção de Raízes e Tubérculos do Instituto Agronômico de Campinas, SP.

0 experimento foi instalado no Centro Experimental de Campinas, do Instituto Agronómico de Campinas, SP, situado à 22.05' Latitude Sul e 47.05' Longitude Oeste a 669 metros de altitude, cujo clima, segundo a classificação de Kb̆ppen, é do tipo Cwa (SETZER, 1966).

o solo do ensaio é classificado como Latossolo roxo, cujas caracteristicas quỉmicas e granulométricas nas profundidades de 0-20, 20-40 e 40-60 cm acham-se na Tabela 4.

Os dados de temperatura média do ar e da distribuição hidrica durante o período estudado e as normais de 20 anos (1956/1976) encontram-se na Tabela 5.

o experimento foi irrigado duas vezes, uma no início de agosto por ocasião da emergência das plantas e a outra um mês após. A finalidade desta operação foi garantir bom "stand" e uniformidade das plantas, em virtude da estiagem que ocorreu neste período (Tabela 5).

Todos os tratamentos foram adubados com a fórmula 40$-80-60 \mathrm{~kg} / \mathrm{ha}$ de $\mathrm{N}, \mathrm{P}_{2} \mathrm{O}_{5}$ e $\mathrm{K}_{2} \mathrm{O}$, respectivamente. O nitrogênio, na forma de sulfato de amônio, foi aplicado somente em cober tura aos 60 dias após a brotação. O fósforo e o potássio, naśs formas de superfosfato simples e cloreto de potássio, respec 


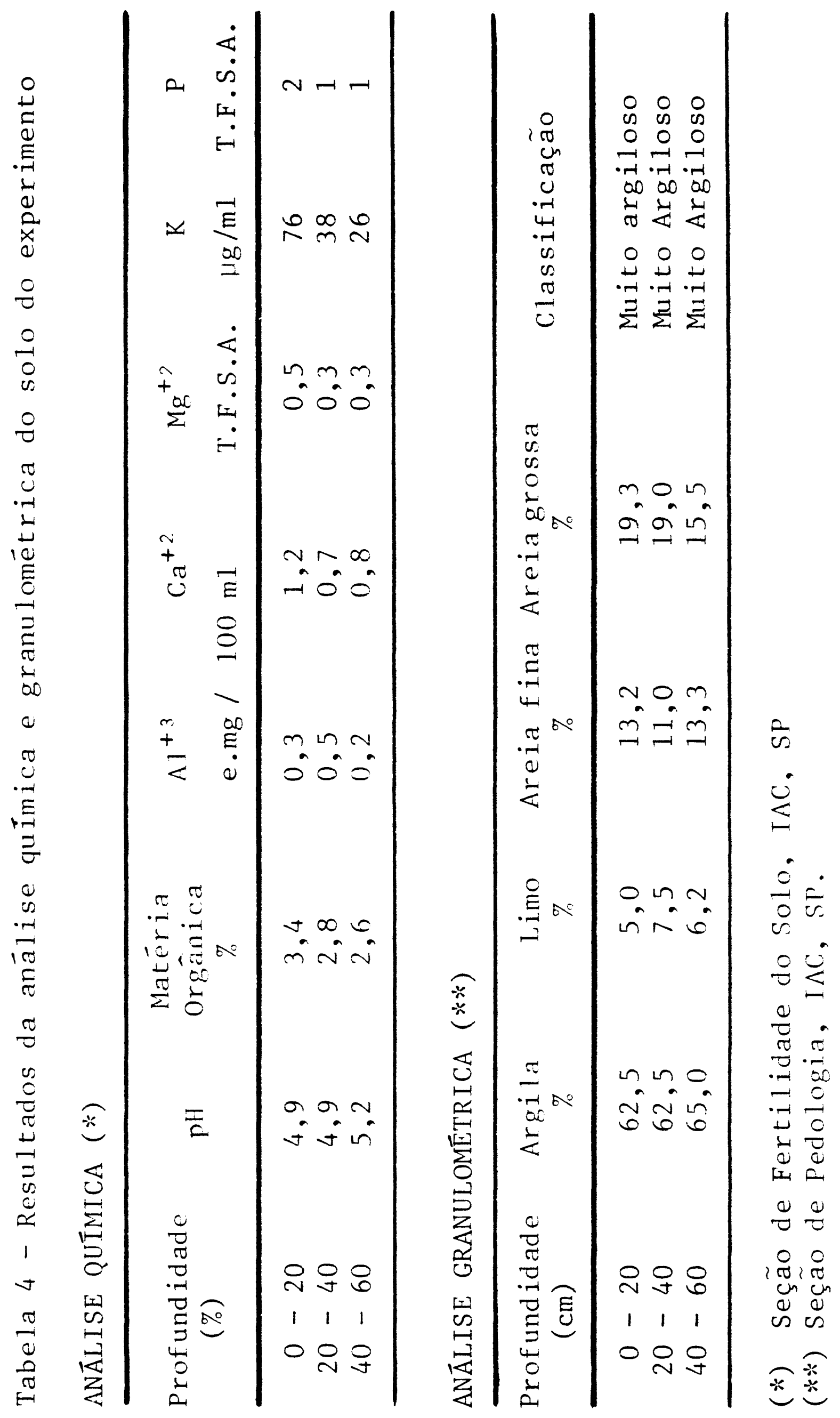


tivamente, foram aplicados nos sulcos de plantio e misturados com a terra.

Tabela 5 - Temperatura média do ar e distribuição hỉdrica no Centro Experimental de Campinas, IAC, SP, nos meses de execução do experimento (*)

\begin{tabular}{|c|c|c|c|c|}
\hline \multirow[b]{2}{*}{ Meses } & \multicolumn{2}{|c|}{ Precipitação natural } & \multicolumn{2}{|c|}{ Temperatura } \\
\hline & $\begin{array}{c}\text { Media de } \\
20 \text { anos } \\
\text { (mm) }\end{array}$ & $\begin{array}{c}75 / 76 \\
(\mathrm{~mm})\end{array}$ & $\begin{array}{c}\text { Media de } \\
20 \text { anos } \\
(\text { ( C) }\end{array}$ & $\begin{array}{c}75 / 76 \\
(9 \mathrm{C})\end{array}$ \\
\hline Julho & 33,4 & 39,4 & 17,2 & 15,7 \\
\hline Agosto & 35,0 & 0,0 & 18,9 & 21,3 \\
\hline Setembro & 57,4 & 23,9 & 20,7 & 21,5 \\
\hline Outubro & 139,0 & 214,9 & 21,2 & 21,0 \\
\hline Novembro & 136,9 & 252,2 & 21,8 & 21,2 \\
\hline Dezembro & 214,0 & 181,0 & 22,5 & 23,1 \\
\hline Janeiro & 240,5 & 271,5 & 23,1 & 23,1 \\
\hline Fevereiro & 216,0 & 276,4 & 23,0 & 21,6 \\
\hline Março & 132,8 & 166,6 & 22,6 & 22,5 \\
\hline Abril & 61,3 & 84,7 & 20,8 & 20,4 \\
\hline Maio & 50,3 & 151,1 & 18,2 & 17,8 \\
\hline Junho & 44,9 & 86,1 & 17,2 & 16,5 \\
\hline Ju 1ho & - & 138,7 & - & 16,1 \\
\hline Agosto & - & 105,3 & - & 18,3 \\
\hline Setembro & - & 182,3 & - & 18,3 \\
\hline
\end{tabular}

(*) Seção de Climatologia Agrỉcola, IAC, SP.

As manivas de ambos os cultivares provieram da Estação Experimental de Piracicaba, SP, as quais receberam os mesmos tratos culturais e apresentavam-se sadias e com cerca de um ano de idade. Foram cortadas com serra circular no tamanho de $25 \mathrm{~cm}$ e plantadas pelo sistema comum, horizontalmente, no es paçamento de $1,0 \times 0,6 \mathrm{~m}$. As parcelas foram constituídas de 108 plantas cada, compostas de 9 linhas de 12 plantas. 
As amostragens das plantas foram feitas em 6 épocas do desenvolvimento, colhendo-se 3 plantas inteiras por repetição, devidamente circundadas por plantas competitivas. A pri preira amostragem foi feita 60 dias após a brotação e as outras a intervalos de 60 dias até o fim do primeiro ciclo vegetativo.

Após a coleta, as plantas foram separadas nas partes: folhas (foliolos + pecíolos), hastes e raizes tuberosas. As amostras foram pesadas, lavadas, secas e moídas, segundo SARRUGE \& HAAG (1974).

As análises químicas foram efetuadas na Seção de Quỉmi ca Analitica do Instituto Agronômico, Campinas, SP, de acordo com os seguintes métodos: $\mathrm{N}$ e $\mathrm{P}$ pelo Autoanalisador II TECHNICON, a partir de amostras diferidas segundo CONCON \& SOLTESS (1973), sendo o $\mathrm{P}$ pelo método colorimétrico do ácido fosfovanadimolíbdico - amarelo (LOTT et alii, 1956) e o $\mathrm{N}$ pelo fenol alcalino (GEHRKE et alii, 1973); S pelo Autoanali sador II TECHNICON segundo TEIXEIRA et alii (1976); Ca e Mǵ (BATAGLIA \& GALLO, 1972) e K (PERKIN-ELMER, 1971).

O delineamento estatístico adotado foi o de blocos ao acaso com 2 (dois) tratamentos e 4 (quatro) repetições, dispostos em parcelas subdivididas para 6 (seis) épocas de coTheita (GOMES, 1973).

\section{RESULTADOS E DISCUSSÃO}

\section{Matéria seca}

A Tabela 6 mostra a produção de matéria seca pelos dois cultivares.

Verificou-se pela anālise estatistica que houve diferença significativa na produção de matéria seca das raízes, sendo o cultivar Mantiqueira mais produtivo que o outro. A in teração cultivar $x$ época também foi significativa ao nivel dē $5 \%$ de probabilidade o que indica que os cultivares comportaram-se diferentemente em relação às épocas de acumulação de matéria seca nas raỉzes. Quando se considera a planta intei- 
ra, não se observa diferença entre os cultivares; isto ocorreu, provavelmente, porque o cultivar Branca de Santa Catari na produziu mais parte aérea (hastes + folhas) que o Manti= queira, compensando a diferença na produção de raízes.

Tabela 6 - Acumulação de matéria seca (*)

\begin{tabular}{|c|c|c|c|c|}
\hline \multirow{2}{*}{$\begin{array}{l}\text { Dias apōs a } \\
\text { brotação }\end{array}$} & \multicolumn{2}{|c|}{ Branca de Sta. Catarina } & \multicolumn{2}{|c|}{ Mantiqueira } \\
\hline & $\mathrm{g} / \mathrm{p} 1$ & $\mathrm{~kg} / \mathrm{ha}$ & $\mathrm{g} / \mathrm{p} 1$ & $\mathrm{~kg} / \mathrm{ha}$ \\
\hline \multicolumn{5}{|l|}{300} \\
\hline Raiz & - & - & 485,3 & 8.088 \\
\hline Haste & - & - & 309,5 & 5.158 \\
\hline Folhas & - & - & 3,4 & 57 \\
\hline Total & - & - & 708,2 & 13.303 \\
\hline \multicolumn{5}{|l|}{360} \\
\hline Raiz & 397,4 & 6.623 & - & - \\
\hline Haste & 419,7 & 6.995 & - & - \\
\hline Folhas & 1,4 & 23 & - & - \\
\hline Total & 818,5 & 13.641 & - & - \\
\hline
\end{tabular}

(*) 4 repetições; população de 16.666 plantas/ha.

A mandioca, à medida que cresce, perde gradativamente suas folhas em função de fatores ambientais e fisiológicos (MENDES, 1940; COURS, 1951; VIEGAS, 1976). Nas condições do Estado de São Paulo, ela chega a ficar totalmente desfolhada na época mais fria e seca do ano. Em virtude da queda gra dativa das folhas, torna-se dificil avaliar a produção rea de matéria seca.

\section{Nutrientes}

As Tabelas 7 e 8 dão os teores de macronutrientes e as quantidades encontradas por planta e estimadas por hectare nos diferentes órgãos analisados. 


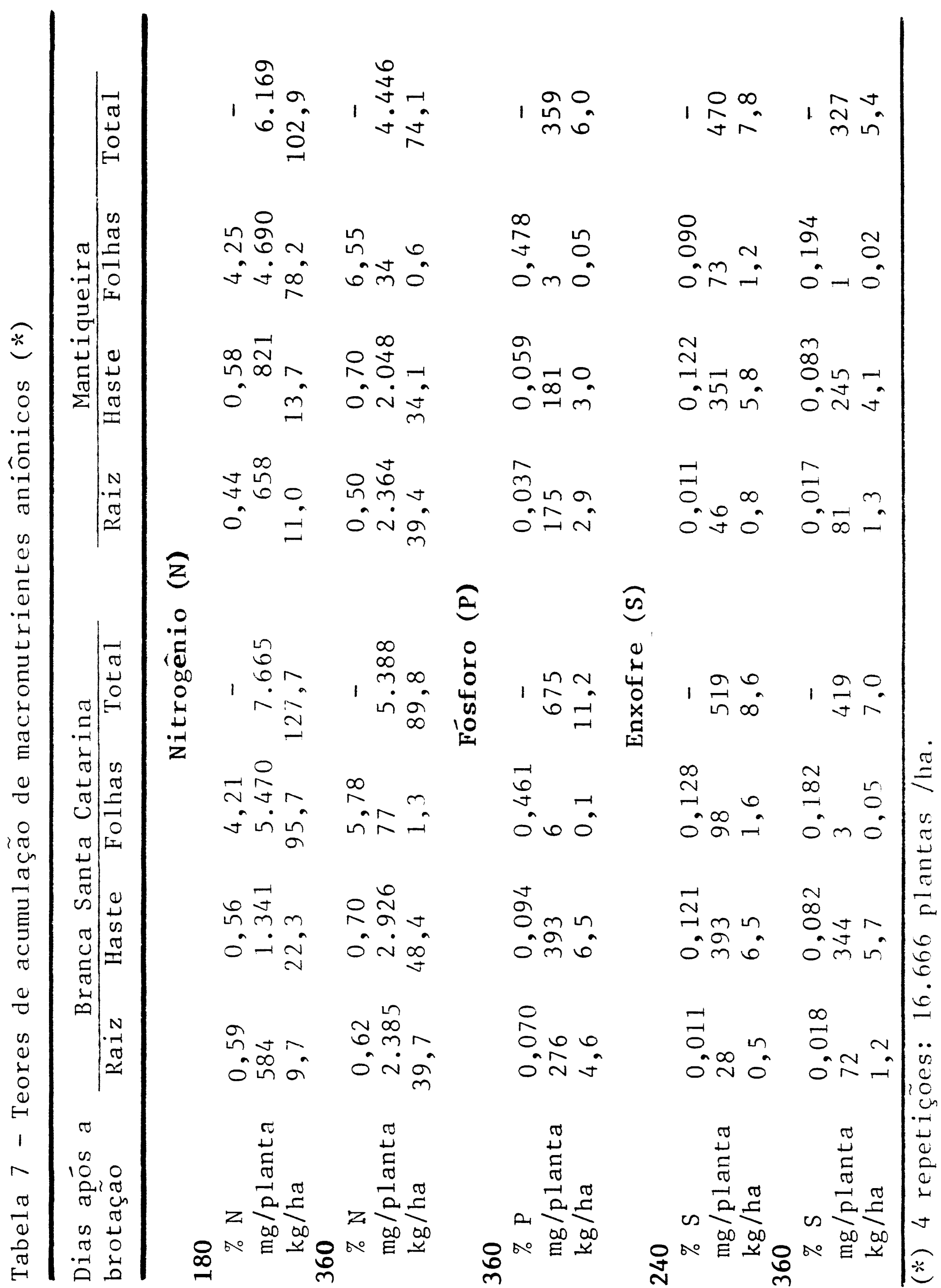




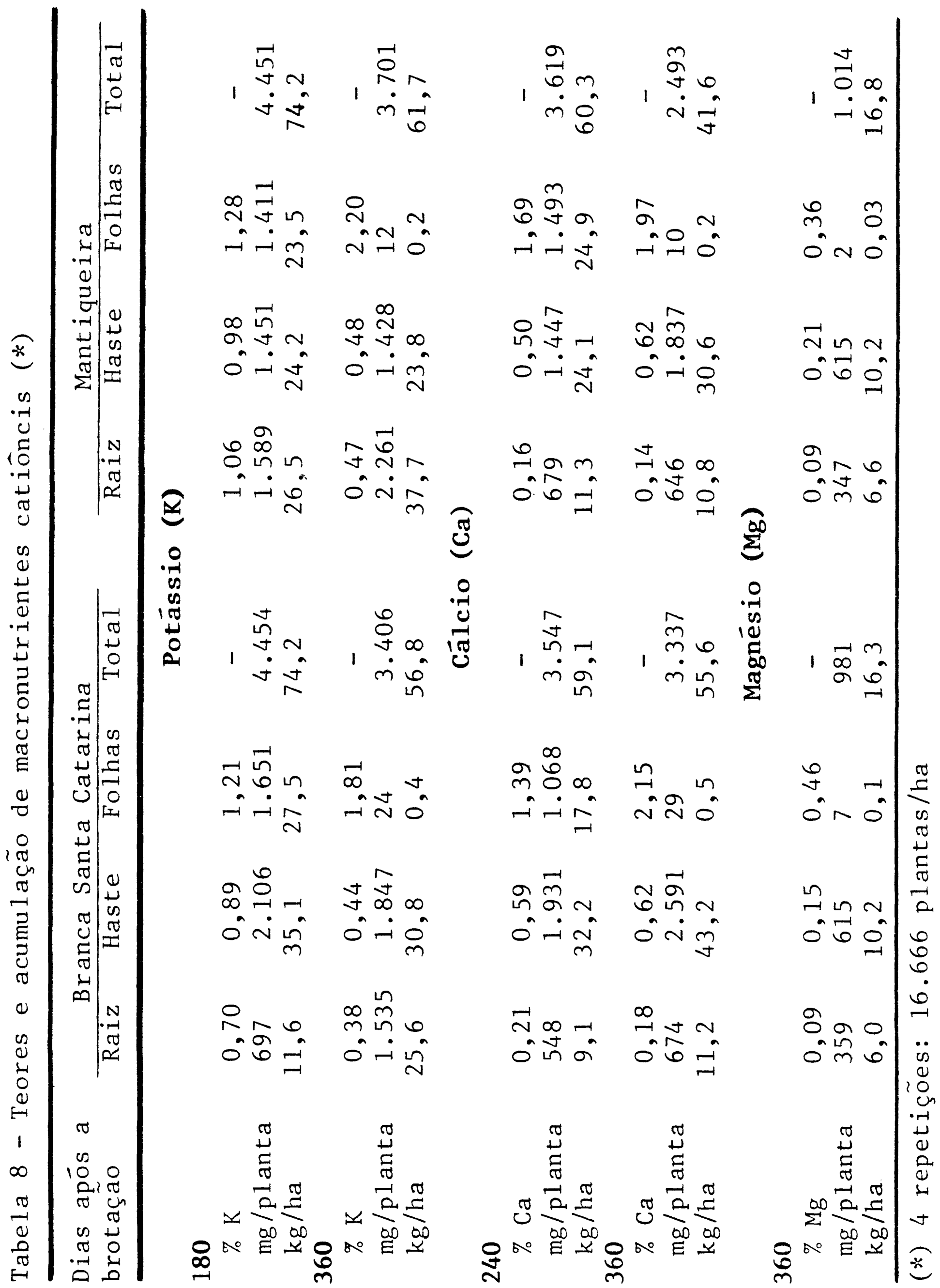


No caso do $\mathrm{N}, \mathrm{K}, \mathrm{Ca}$ e $\mathrm{S}$, verifica-se que as quantidades existentes aos 360 dias são menores do que os achados em outro período que não foi o mesmo para esses 4 elementos: is to deve ser em grande parte devido à perda de folhas, confor me já discutido.

Embora o cv. Branca de Santa Catarina tivesse acumulado quase $20 \mathrm{~kg}$ de $\mathrm{N} / \mathrm{ha}$ mais que o Mantiqueira, a diferença não foi significativa. No caso do $P$, porém, $O \mathrm{cv}$. Mantiqueira foi significativamente menos exigente.

Quando se considera a planta inteira, os cultivares não diferem no total de $\mathrm{K}$ acumulado, provavelmente, pela maior quantidade de $K$ na parte aérea (hastes + folhas) do cultivar Branca de Santa Catarina.

De acordo com a literatura consultada, nota-se que o $\mathrm{K}$, em média, é o elemento mais absorvido pela mandioca (Tabe la 1).

Pelos dados apresentados, a acumulação foi maior para - N seguido pelo $\mathrm{K}$, o que, apesar de não concordar com a maio ria dos autores, está de acordo com OELSLIGLE (1975), DUFOUR NET \& GOARIN (1957) e BONNEFOY (1933).

Pelos resultados apresentados, verifica-se que a quan tidade de cálcio absorvida é alta, vindo logo após o potássio, sendo necessário mais estudos para que a questão seja me thor esclarecida.

A tolerância da mandioca à acidez do solo é citada com frequência na literatura. O efeito benéfico da calagem, normalmente, é atribuído ao fornecimento de cálcio e magnésio do que propriamente à elevação do $\mathrm{pH}$ ou à neutralização do aluminio (HOWELER, 1975).

Por essa razão e, em virtude das quantidades razoáveis de cálcio existentes nos solos, raramente tem se conseguido efeito positivo da calagem.

HOWELER (1975), trabalhando em oxissolos da Colômbia, parecidos com os solos de cerrado do Brasil, conseguiu res- 
posta à calagem (relação $\mathrm{Ca}: \mathrm{Mg}$ de $10: 1$ ) na dose máxima de 0,5 $t / h a$. Além dessa dose, o rendimento caiu. Segundo o autor, es sa queda pode ser devida à diminuição na absorção de $\mathrm{K}, \mathrm{Zn}$, $\mathrm{Cu}$ e $\mathrm{Mn}$, elementos estes, que a mandioca parece exigir mais que os outros cultivos ou não tem a mesma capacidade para extrai-los.

No Estado de São Paulo, a mandioca não tem respondido satisfatoriamente à calagem, mesmo em solos pobres é ácidos.

A acumulação do enxofre nas raizes foi maior para o cultivar Mantiqueira e menor quando se considerou a planta in teira. Esta inversão, provavelmente, é devida à participação diferencial da matéria seca dos cultivares, uma vez que a concentração de enxofre nos órgãos foi semelhante para ambas.

0 enxofre tem sido muito pouco estudado em mandioca. Ngongi (1976), citado por HOWELER (1978), encontrou nas planícies orientais da Colômbia, resposta positiva ao sulfato de potássio em relação ao cloreto de potássio e obteve efeito similar misturando enxofre com ácido clorídrico. 0 autor con cluiu que o enxofre era fator limitante e que aplicações al= tas de cloretos podiam inibir a absorção de sulfato e induzir deficiência.

As Figuras 1 e 2 dão, respectivamente as quantidades de macronutrientes extraidas e exportadas quando se consideram as produções de raiz observadas no ensaio e uma colheita de 1 tonelada de raíz fresca. Verificou-se que os elementos se dispõem segundo a ordem decrescente:

\section{extração}

Branca de Santa Catarina - N $\mathrm{K} \quad \mathrm{Ca} \quad \mathrm{Mg} \quad \mathrm{P} \quad \mathrm{S}$ $\begin{array}{lllllll}\text { Mantiqueira } & -\mathrm{N} & \mathrm{K} & \mathrm{Ca} & \mathrm{Mg} & \mathrm{P} & \mathrm{S}\end{array}$

\section{exportação (raízes)}

Branca de Santa Catarina - N $\mathrm{K}$ Ca $\mathrm{Mg} \quad \mathrm{P} \quad \mathrm{S}$

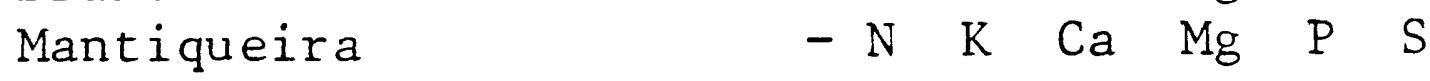




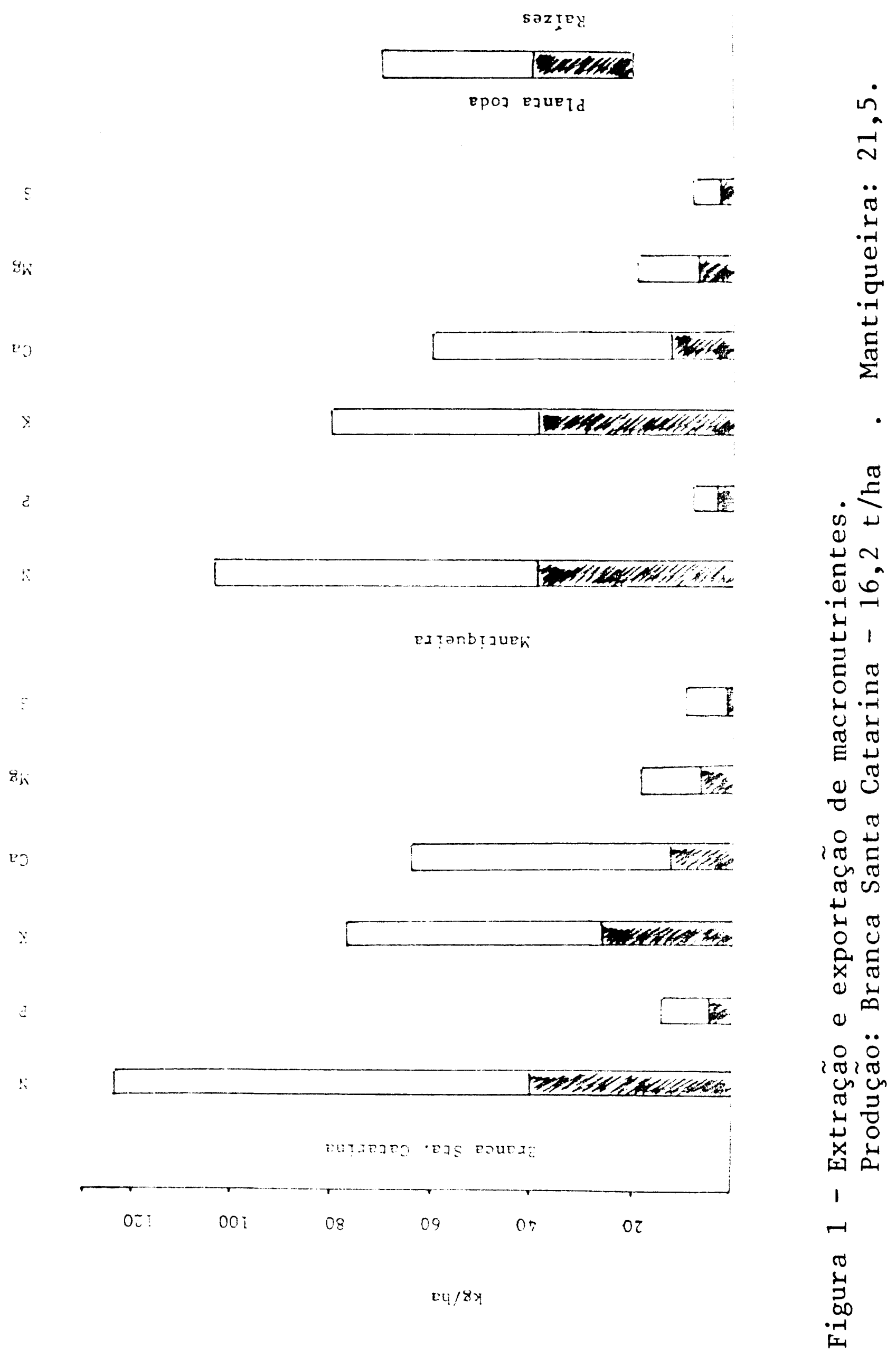


$s$

8 is

ej

$x$

d

N

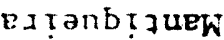

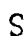

$8 \mathrm{~W}$

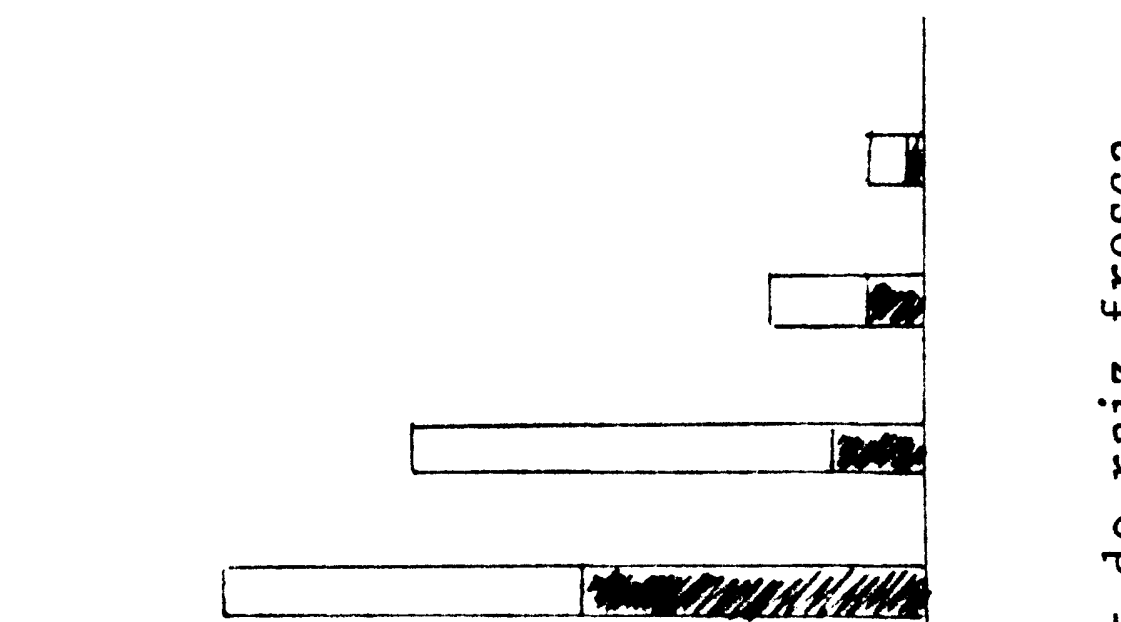

$\mathrm{S}$

is

$x$

d
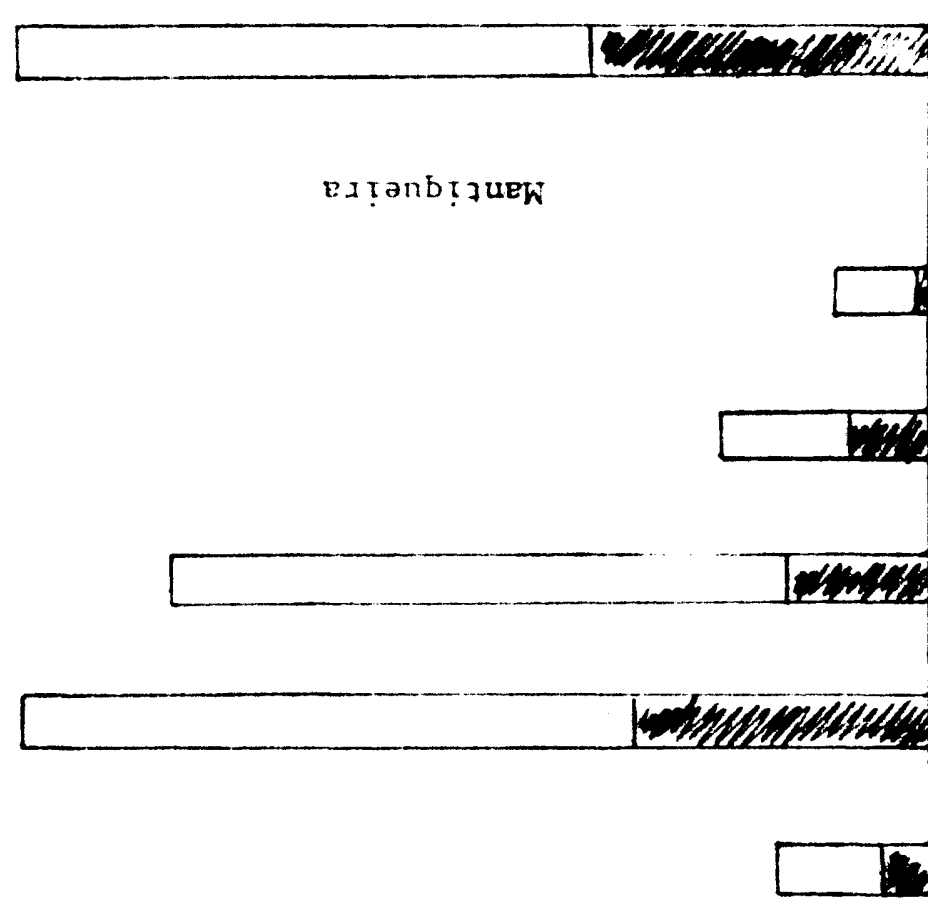

0
0
0
0
4
4
$N$
0
0
0
0
0

$\downarrow$

岁

क

$\stackrel{+}{ت}$

$\cdot \sqrt{7}$

峞

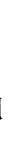

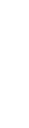

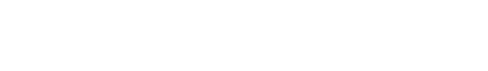

s

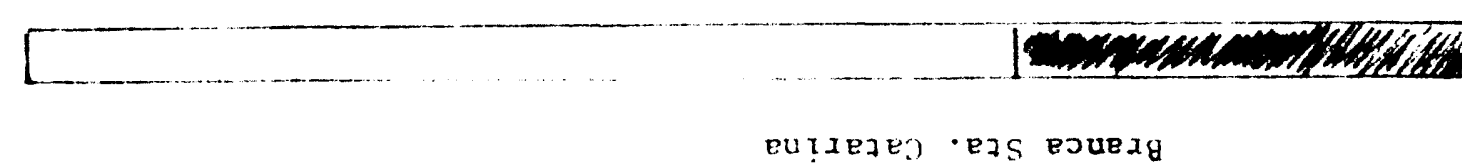

eutaejej • ezs bouedg

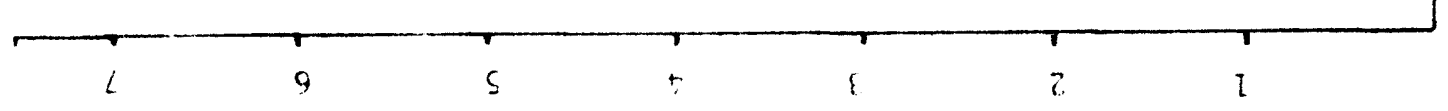

$3 / 84$

$1 \stackrel{0}{0}$

帒

1

$\sim$

茫 


\section{RESUMO E CONCLUSÕES}

0 presente trabalho teve por objetivo verificar a produção de matéria seca, extração e exportação dos macronutrientes e produção de raízes nos cultivares de mandioca Bran ca de Santa Catarina e IAC Mantiqueira.

0 experimento foi instalado em ärea do Centro Experimental de Campinas, IAC, SP, no ano agrícola 1975/76, em so1o de grande grupo Latossolo Roxo.

Utilizou-se uma adubação $\mathrm{N}-\mathrm{P}_{2} \mathrm{O}_{5}-\mathrm{K}_{2} \mathrm{O}$ de 40-80-60 kg/ ha. O nitrogênio, na forma de sulfato de amônio, foi aplicado somente em cobertura aos 60 dias após a brotação. O fósfó ro e o potássio, na forma de superfosfato simples e cloreto de potássio, respectivamente, foram aplicados no fundo dos sulcos por ocasião do plantio.

0 experimento foi irrigado duas vezes, aos 15 e 45 dias após o plantio, com a finalidade de se obter bom "stand" e uniformidade das plantas, em virtude da estiagem que ocorreu neste periodo.

As plantas amostradas foram divididas em raizes, hastes e folhas e analisadas para N, P, K, Ca, Mg e S.

0 delineamento adotado foi o de blocos ao acaso. As cur vas representativas da acumulação da matéria seca e nutrien= tes, pela planta inteira e pelas raízes, foram obtidas a partir dos dados calculados por equações de regressão. As quantidades extraídas de nutrientes foram calculadas através do ponto de máximo destas equações.

As principais conclusões e os dados mais relevantes fo ram os seguintes:

- Houve diferença estatística na produção de raỉzes dos cultivares;

- A extração de macronutrientes foi a mesma para os dois cultivares, exceto para o P. A exportação foi di ferente somente para o $\mathrm{K}$ e $\mathrm{S}$. 


\section{SUMMARY}

MACRONUTRIENT REQUIREMENTS BY TWO CASSAWA CULTIVARS (Manihot esculenta CRANTZ).

This paper deals with the results of a field experiment conducted in order to study dry matter production and macronutrient accumulation in two cassava cultivars 'Branca de San ta Catarina' and 'IAC Mantiqueira'.

$\mathrm{Plants}$ received an uniform dressing of $\mathrm{N}, \mathrm{P}_{2} \mathrm{O}_{5}$ and $\mathrm{K}_{2} \mathrm{O}$ of 40,80 and $60 \mathrm{~kg} / \mathrm{ha}$, respectively as ammonium sulphate, simple superphosphate, and muriate of potassium; $N$ was top dressed 60 days after emergence.

Two irrigations were provided in the beginning of the growth cycle.

Plants were sampled and analysed for growth parameters and macronutrient composition in six occasions with 60 day intervals :

The main conclusions and relevant data were as follows:

1. there was a statistical difference in root production of the two cultivars; maximum dry matter accumulation took place in the period pf $120-180$ days after emergence;

2. extraction of macronutrients was the same in the two cultivars, except for $P$; export was different only in the case of $\mathrm{K}$ and $\mathrm{S}$.

LITERATURA CITADA

BATAGLIA, O.C.; GALLO, J.R., 1972. Determinação de cálcio e de magnésio em plantas por fotometria de chama de absorção. Bragantia 31: 59-74.

BONNEFOY, J.V., 1933. Calcul des éléments fertilizants enlevés au sol par une récolte de manioc. Bulletin Économique Madagascar (83): 75-77. 
CATANI, R.A.; GALLO, J.R.; GARGANTINI, H., 1954. Extração de elementos nutritivos do solo por diversas culturas. Campi nas, Instituto Agronómico (Cartaz).

CONCON, J.M.; SOLTESS, D., 1973. Rapid micro Kjeldah1 digestion of cereal grains and other biological materials. Analytical Biochemistry 53(1): 35-41.

COURS, G., 1951. Le manioc à Madagascar. Mémoires de l'Insti tut Scientifique de Madagascar. Sér. B.: Biologie Végétale, Tananarive, 3(2): 203-416.

COURS, G., 1953. Le diagnostic foliaire et les carences. Recherches Agronomique de Madagascar. Compte Rendu Tananari ve (2): 78-84.

DE GEUS, J.G., 1967. Root crops: cassava. In: Fertilizer guide for tropical and subtropical farming, Zurich, Centre D'Etude de 1'Azote, p.181.185.

DUFOURNET, R.; GOARIN, P.. 1957. Note sur la culture du manioc à Madagascar. Riz et reziculture et cultures vivrié res tropicales 3(1): 15-38.

DULONG, R., 1971. Le manioc a Madagascar. Agronomie tropicale 26(8): 791-829.

FAO, 1975. Production Yearbook, Rome, vol. 29.

FOX, R.H.; TALLEYRAND, H.; SCOTT, T.W., 1975. Effect of nitrogen fertilization on yields and nitrogen contend of cassava, Llanera cultivar. Journal of Agriculture of the University of Puerto Rico 59(2): 115-124.

FUNDAÇÃO IBGE, 1975. Anuário Estatístico do Brasil, Rio de Janeiro, Vol. 36, p.167.

GEHRKE, C.W.; WALL, L.L.; ABSHEER, J.S., 1973. Automated nitrogen method for fields. Jour, Assoc. Off. Agric. Chemists 56(5): 1096-1105. 
GOMES, F.P., 1973. Curso de Estatística Experimental, 7.a ed São Paulo, Editora Nobel, 430p.

HONGSAPAN, S., 1962. Does planting of cassava really impove rish the soil? Kasikorn 35(5): 403-407 (apud HOWELER, R. H., 1978).

HOWELER, R.H., 1975. Requirmento de elementos secundários e elementos menores da mandioca. In: Curso Especial de Aperfeiçoamento para pesquisadores de mandioca, Cali, Colômbia, CIAT, $14 \mathrm{p}$.

HOWELWE, R.H., 1978. Nutrición mineral y fertilización de la yuca. In: Curso de producción de yuca, Cali, Colômbia, CIAT, vol. 1, p.274-321.

KANAPATHY, K; KEAT, G.A., 1970. Growing maize, sorghum and tapioca on peat soil. In: Proceedings of the Conference on Crop Diversification in Malaysian, Kuala Lampur. p.2535 .

KANAPATHY, K., 1974. Fertilizer experiement on shallow peat under continuous cropping with tapioca. The Malaysian agric. Jour. 49(4): 403-412.

LOTT, W.L.; NERY, J.P.; GALLO, J.R.; MEDCALF, J.C., 1956. A técnica de análise foliar aplicada ao cafeeiro, Campinas, Instituto Agronômico, 29p. (Boletim, 79).

MEJIA, F.R., 1946. El cultivo de la yuca, y su Explotación industrial. Agricultura tropical 2(3): 13-21 (apud HOWELER, R.H., 1978).

MENDES, C.T., 1940. Contribuição para o estudo da mandioca, São Paulo, Secretaria da Agricultura, Indústria e Comércio, 99 p.

NESTEL, B., 1973. Current utilization and future potential for cassava. In: Proceedings of Chronic Cassava Toxicity, London, p.11-26.

NGONGI, A.G.N., 1976. Influence of some mineral nutrients on 
growth, composition and yield of cassava (Manihot esculen ta Crantz). Ithaca, Cornell Un. (Ph.D. Thesis) (apud HOWELER, R.H., 1978).

NIJHOLT, J.A., 1935. Opname van voendingsstoffen uit den boden buj cassave. Algemeen Proefstation voor den Landbouw Buitenzorg, Korte Mededeelingen n? 15 (apud HOWELER, R.H., 1978).

OELSLIGLE, D.D., 1975. Accumulation of dry matter, nitrogen, phosphorus, and potassium in cassava. Turrialba 25(1): 8587.

PERKIN-ELMER CORPORATION, 1971. Analytical methods for atomic absorption spectriphotometry, Norwalk, Connecticut.

SARRUGE, J.R.; HAAG, H.P., 1974. Análise química em plantas, Piracicaba, ESALQ-USP, 52p.

SETZEN, J., 1966. Atlas climático e ecológico do Estado de São Paulo, São Paulo, Instituto Geológico e Geográfico, p. 35-38.

SOLORZANO, N.; BORNEMISZA, E., 1976. Estudios del cultivo de yuca en Costa Rica. II. Composición química y producción de três cultivares. Turrialba 26(3): 261-264.

TEIXEIRA, J.P.F. et alii, 1976. Determinação automatizada de enxofre em plantas, pelo sistema auto-analisador II Technicon. Bragantia 35(1): LXXVII-LXXXI.

VELLY, J., 1969. Contribution à la determination de la fumure d'entretien; les exportations en elements mineraux de principales cultures. Bulletin de Madagascar 19(282): 872 -890 (apud HOWELER, R.H., 1978).

VIEGGAS, A.P., 1976. Estudos sobre a mandioca, São Paulo. IAC Brascan Nordeste, 214p. 